\title{
Search for cosmic-ray antiproton origins and for cosmological antimatter with BESS
}

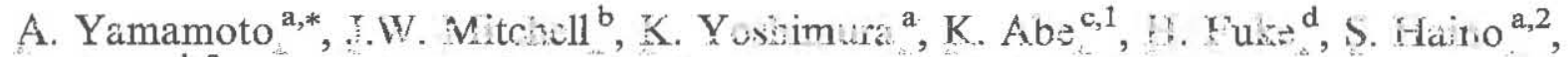

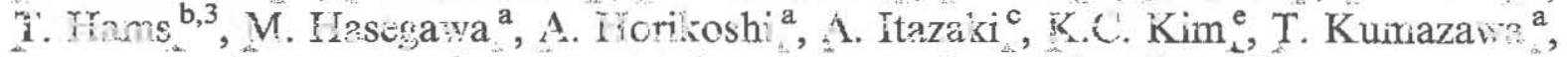

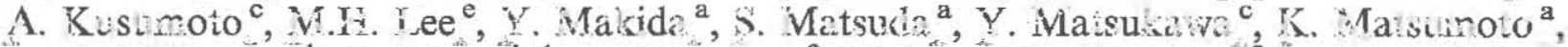

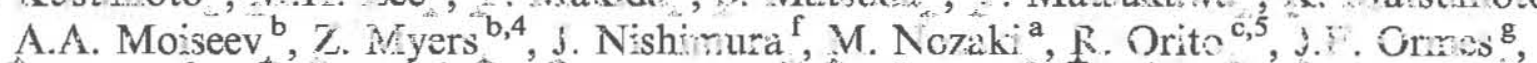

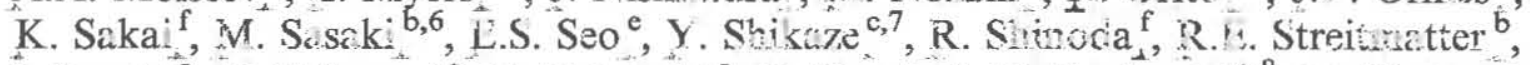

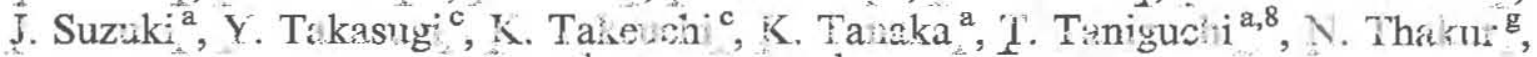 \\ T. Yamagami ${ }^{\text {d }}$ T. Yoshicla ${ }^{\mathrm{d}}$, BESS Collaboration \\ ${ }^{a}$ High Energy Accelerator Research Organization (KEK), Tsukuba, Ibaraki 305-0801, Japan \\ ${ }^{\mathrm{b}}$ National Aeronautics and Space Administration, Goddard Space Flight Center (NASA/GSFC), Greenbelt, MD 2077I, USA \\ ${ }^{\mathrm{C}}$ Kobe University, Kobe, Hyogo 657-8501, Japan \\ dInstitute of Space and Astronautical Science, Japan Aerospace Exploration Agency (ISASIJAXA), Sagamihara, Kanagawa 229-8510, Japan \\ ${ }^{\mathrm{e}}$ IPST, University of Maryland, College Park, MD 20742, USA \\ ${ }^{\mathrm{f}}$ The University of Tokyo, Bunkyo, Tokyo 113-0033, Japan \\ ${ }^{g}$ University of Denver, Denver, CO 80208 , USA
}

\section{Abstract}

The balloon-borne experiment with a superconducting spectrometer (BESS) has performed cosmic-ray observations as a US-Japan cooperative space science program, and has provided fundamental data on cosmic rays to study elementary particle phenomena in the early Universe. The BESS experiment has measured the energy spectra of cosmic-ray antiprotons to investigate signatures of possible exotic origins such as dark matter candidates or primordial black holes, and searched for heavier antinuclei that might reach Earth from antimatter domains formed in the early Universe. The apex of the BESS program was reached with the Antarctic flight of BESS-Polar II, during the 2007-2008 Austral Summer, that obtained over 4.7 billion cosmic-ray events from 24.5 days of observation. The flight took place at the expected solar minimum, when the sensitivity of the low-energy antiproton measurements to a primary source is greatest. Here, we report the scientific results, focusing on the long-duration flights of BESS-Polar I (2004) and BESS-Polar II (2007-2008). (C) 2011 Published by Elsevier Ltd. on behalf of COSPAR.

Keywords: Cosmic rays; Antiproton; Antimatter; Primordial blaci hole; Dakk Antarticis Scientific balloon; BESS;

\footnotetext{
* Corresponding author. Tel.: +8129864 5459; fax: +81298643209.

E-mail address: akira.yamamoto@kek.jp (A. Yamamoto).

${ }^{1}$ Present address: ICRR, The University of Tokyo, Kamioka, Gifu 506-1205, Japan.

${ }^{2}$ Present address: Instituto Nazionale di Fisica Nucleare (INFN), Perugia, Itely.

${ }^{3}$ Also University of Maryland Baltimore County, Baltimore, MD, USA.

${ }^{4}$ Present address: Physics Department, Technion - Israel Institute of Technology, Technion City, Haifa, Israel.

${ }^{5}$ Present address: Tokushima University, Tokushima, Japan.

6 Also University of Maryland, College Park, MD, USA.

${ }^{7}$ Present address: Japan Atomic Energy Agency (JAEA), Tokai, Ibaraki, Japan.

${ }^{8}$ Present address: Okayama University, Okayama, Japan.
} 


\section{Introduction}

Progress in modern observational cosmology and astrophysics has shown that the material Universe is dominated by dark matter responsible for the formation of structure and for the dynamics of galaxies. The nature of the dark components, however, is unknown. Similarly, it is observed that cosmological antimatter is apparently absent in the present era, but the reason for this absence remains as a major problem for cosmology and particle physics. It has been suggested that one constituent of the dark matter may be primordial black holes (Hawking, 1975; Barrau et al., 2002), formed in the early Universe due to the collapse of dense regions formed by density fluctuations. The detection of $\mathrm{PBH}$ through antiparticles arising from the Hawking radiation emitted as they evaporate would probe the early Universe at very small scales (Maki et al., 1996). PBH evaporation might be detected by its effect on the measured antiproton spectrum. Addressing these issues are central scientific goals of the BESS program (Yoshimura, 2001; Yamamoto et al., 2008; Mitchell et al., 2009). The precise measurements of the low-energy cosmic-ray antiproton flux and the sensitive search for heavier antinuclei made by the BESS experiment are vital to constraining candidate models for dark matter, evaluating the possible density of primordial black holes, and seeking for the limits of cosmological antimatter. BESS also provides important fundamental data on the spectra of light cosmic-ray elements and isotopes and for studies of the effect of the out-flowing solar wind on the Galactic cosmic rays (Mitchell et al., 2009). The exceptionally large collecting power and precise particle identification capability of the BESS instruments enable a broad scientific reach.

BESS uses a superconducting magnetic-rigidity spectrometer with a time-of-flight (TOF) system and an aerogel Cherenkov counter (ACC) to fully identify incident particles by charge, charge sign, rigidity, and velocity (Ajima et al., 2000; Yoshida et al., 2004). The joint US-Japan BESS program, supported by NASA and ISAS-JAXA, carried out eleven successful balloon flights from 1993 to
2008 , nine approximately one-day northern-latitude flights and two long-duration Antarctic flights, as summarized in Table 1 . These have collectively recorded more than 13,000 cosmic-ray low-energy antiprotons and set the most stringent upper limits to the existence of antihelium and antideuterium. BESS has also provided the reference standard for elemental and isotopic spectra of $\mathrm{H}$ and $\mathrm{He}$ over more than a full solar cycle. Together with the antiproton measurements, these provide strong constraints on models of cosmic-ray transport in the Galaxy and Solar System.

\section{Progress of the BESS and BESS-Polar experiments}

The BESS program began as an outgrowth of work toward the Astromag superconducting magnet facility that was planned for the International Space Station, ISS (Ormes, 1986). From the early 1980s, there was tremendous excitement over results from seminal balloon-borne experiments that reported detecting substantial excesses of antiprotons at both high and low energies using magnetic spectrometers or annihilation signatures. By the mid1980 s, the cosmic-ray community was fully engaged in an effort to measure cosmic ray matter and antimatter to unprecedented precision. During the Astromag study, a number of magnet configurations were proposed. BESS stemmed from a proposal to use a solenoidal superconducting magnet with a coil thin enough for particles to pass through with minimal interaction probability (Yamamoto et al., 1988). This configuration maximizes the opening angle of the instrument, and hence the geometric factor, making it ideal for rare-particle measurements. BESS began as a balloon-borne instrument to validate this concept, and rapidly evolved into an immensely capable scientific program in its own right (Orito, 1987).

The BESS instruments consist of thin superconducting solenoidal magnets and high-resolution detector systems. For energies between about $0.1 \mathrm{GeV}$ and $4 \mathrm{GeV}$, referenced to the top of the atmosphere (TOA), the BESS instruments accurately identify incident particles by directly measuring
82

71

Table 1

Progress of the BESS and BESS-Polar balloon flights and observations.

\begin{tabular}{|c|c|c|c|c|c|c|c|c|c|c|c|}
\hline & 1993 & 1994 & 1995 & 1997 & 1998 & 1999 & 2000 & 2001 & 2002 & 2004 & 2007 \\
\hline Location & Canada & $\gg$ & $\gg$ & 2 & $\gg$ & $\gg$ & $\gg$ & US & C. & Ant. & Ant. \\
\hline Float time (h) & 17.5 & 17 & 19.5 & 20.5 & 22.0 & 34.5 & 44.5 & 1.0 & 16.5 & 205 & 730 \\
\hline Observation time, float (h) & 14 & 15 & 17.5 & 18.3 & 20.0 & 31.3 & 32.5 & 1 & 11.3 & 180 & 588 \\
\hline Observation time, asc./des. (h) & & & & & & 2.8 & 2.5 & 12.8 & 2.3 & 3.3 & 3.5 \\
\hline Recorded events $\left(\times 10^{6}\right)$ & 4.0 & 4.2 & 4.5 & 16.2 & 19.0 & 19.1 & 17.0 & N/A & 13.7 & 900 & 4700 \\
\hline Data volume (GB) & 4.5 & 6.5 & 8.0 & 31 & 38 & 41 & 38 & N/A & 56 & 2,140 & 13,500 \\
\hline Event filtering & Yes & Yes & Yes & Yes & Yes & Yes & Yes & Yes & Yes & No & No \\
\hline $\operatorname{MDR}(\mathrm{GV})$ & 200 & 200 & 200 & 200 & 200 & 200 & 200 & 1,400 & 1,400 & 240 & 270 \\
\hline TOF resolution (ps) & 300 & 300 & 100 & 75 & 75 & 75 & 75 & 75 & 75 & 160 & 120 \\
\hline $\mathrm{ACC}$ index & - & - & - & 1.03 & 1.02 & 1.02 & 1.02 & 1.02 & 1.02 & 1.02 & 1.03 \\
\hline Antiproton events observed & 6 & 2 & 43 & 415 & 384 & 668 & 558 & N/A & 147 & 1520 & $\sim 8000$ \\
\hline Antiproton's energy (GeV) & .0 .5 & $\because 0.5$ & $<3.6$ & $\leqslant 3.6$ & -3.6 & $\$ 3.6$ & $\cdot \because 4.2$ & N/A & -4.2 & $<4.2$ & $\therefore 3.5$ \\
\hline Anti-He/He upper limit $\left(\times 10^{-6}\right)$ & 22 & 4.3 & 2.4 & 1.4 & 1.0 & 0.8 & 0.68 & N/A & 0.65 & 0.27 & 0.07 \\
\hline
\end{tabular}


their charge, charge-sign, magnetic rigidity, and velocity. This information is subsequently used to derive their mass and kinetic energy. Elemental spectra can be measured to $>100 \mathrm{GeV}$. All BESS instruments, improved during the course of the program, use similar instrument configurations with detail changes reflecting the evolution of the instruments and flight-specific requirements (Yoshida et al., 2004; Yamamoto et al., 2008; Mitchell et al., 2009). Fig. 1 shows a schematic cross-sectional view of the BESS-Polar $I I$ instrument as an example. A central JET-type drift chamber tracking system and inner drift chambers (IDC), giving 52 trajectory points in the bending direction, are located inside the warm bore of the solenoid to measure the trajectories of charged particles as they pass through the magnetic field. The magnetic rigidity, $R=p c /$ $Z e$ (where $p$ is momentum, $c$ is light velocity, and $Z$ is the particle electric charge) is determined by fitting the curvature of the track through the field. The charge-sign of an incident particle is determined by the direction of its curved track with respect to the local vector magnetic field. Arrays of time-of-flight (TOF) scintillation counters (Shikaze et al., 2000) are located at the top (UTOF) and bottom (LTOF) of the instrument. In BESS-Polar, a middle TOF scinillator array (MTOF) is located inside the magnet bore below the lower IDC. The TOF scintillators trigger readout of events and measure $Z$, and velocity, $\beta$, of incident particles. Particie momentum, $p$, is determined from $R$ and $Z$ and in turn, particle mass, $m$, is determined from $p$ and $\beta$. BESS separates antiprotons from negative charge background particles, mainly muons and electrons, by mass up to an energy of about $1.5 \mathrm{GeV}$. Above this energy, an aerogel Cherenkov counter (ACC) identifies low mass, high $\beta$, background particies. Additional background rejection is supplied by multiple measurements of ionization energy loss $(\mathrm{d} E / \mathrm{d} x)$ from the JET. The horizontal cylindrical configuration of the BESS instrument allows a full opening angle of $\sim 90^{\circ}$ with a resulting acceptance of $0.3 \mathrm{~m}^{2} \mathrm{sr}$. The thin solenoid magnet allows the incoming cosmic rays to

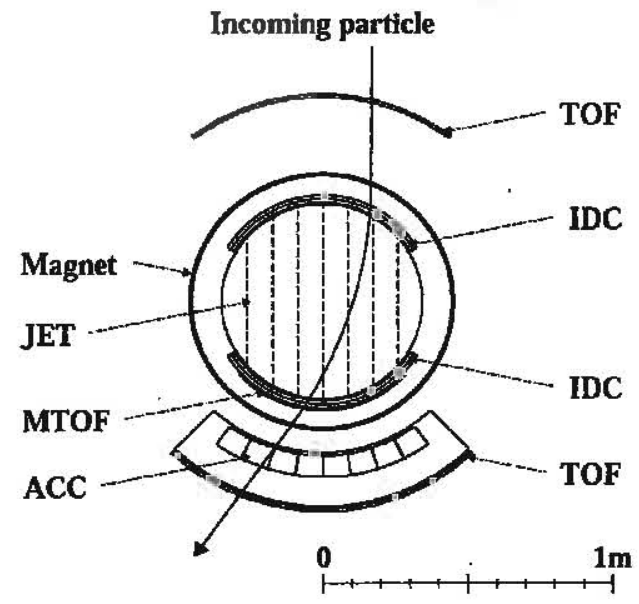

Fig. 1. Cross section of the BESS-Polar II spectrometer. penetrate the spectrometer with minimum interactions (Yamamoto et al., 1988; Makida et al., 2005). Since the magnetic field is very uniform inside the solenoid, the deflection measurement is very accurate for all trajectories within the instrument geometric acceptance. A maximum detectable rigidity (MDR) of $200 \mathrm{GV}$ was achieved in the original BESS instrument and $280 \mathrm{GV}$ in BESS-Polar. For the BESS-TeV flights in 2001 and 2002, outer drift chambers were added to raise the MDR to 1400 GV (Haino et al., 2004).

Versions of the original BESS instrument were used for the initial 9 northern-latitude flights. In order to take advantage of the long flight durations and low geomagnetic cutoff in Antarctic flights, a completely new version of the instrument, BESS-Polar, was developed (Yamamoto et al., 20022; Yoshida et al., 2004; Mitchell et al., 2004; Yoshimura et al., 2008). The BESS-Polar magnet has half the material (radiation) thickness in the coil wall, achieved by use of improved superconducting wire with $\mathrm{Al}$ stabilizer strengthened by alloying with $\mathrm{Ni}$ and by cold-working (Yamamoto et al., 2002b; Makida et al., 2005). Reduced heat transmission to the low-temperature components gives a much improved cryogen lifetime. In addition, the outer pressure vessel was eliminated, the ACC was moved to the bottom, and the MTOF was added. The result was a spectrometer with $\sim 4.5 \mathrm{~g} / \mathrm{cm}^{2}$ encountered by incident triggering particles compared to $\sim 18 \mathrm{~g} / \mathrm{cm}^{2}$ in the previous BESS instrument, lowering the effective energy threshold to well below $100 \mathrm{MeV}$ at TOA. The BESS-Polar data acquisition system has the required throughput and storage capacity to record all triggered events, and so no longer requires down-sampling of proton data. Greatly reduced power consumption and a new solar-cell array power system enable long-duration flights. In BESS-Polar I, the magnet cryogen lifetime was 11 days. BESS-Polar I was flown in 2004, acquiring data for 8.5 days and recording $\sim 2$ terabytes of data on $9 \times 10^{8}$ cosmic ray events. High-voltage breakdown in some of the TOF photomultiplier units reduced the geometric acceptance to about $0.2 \mathrm{~m}^{2} \mathrm{sr}$ and impacted TOF resolution. BESS-Polar I measured 432 antiprotons at energies below $1.3 \mathrm{GeV}$, nearly a 4-fold increase in statistics over BESS measurements during the previous solar minimum, and 1512 antiprotons over the $0.1-4.2 \mathrm{GeV}$ energy range. Technical improvements for BESS-Polar II, see Table 1 , addressed cryogen lifetime, detector performance and stability, power system performance, data storage, and the efficiency of the final prelaunch assembly process. For BESS-Polar II, cryogen lifetime was increased to $>25$ days, the TOF resolution was effectively improved to $\sim 120 \mathrm{ps}$, the rejection power of the ACC was increased to $\sim 6000$, and the full geometric acceptance of $0.3 \mathrm{~m}^{2} \mathrm{sr}$ was maintained throughout the fight. BESS-Polar II operated at float altitude for 24.5 days with the magnet energized, recording 13.5 terabytes of data on over $4.7 \times 10^{9}$ cosmic ray events. This more than doubles the combined data from all previous BESS flights, including BESS-Polar I, and is several times 


JASR 10669
5 August 2011 Disk Used

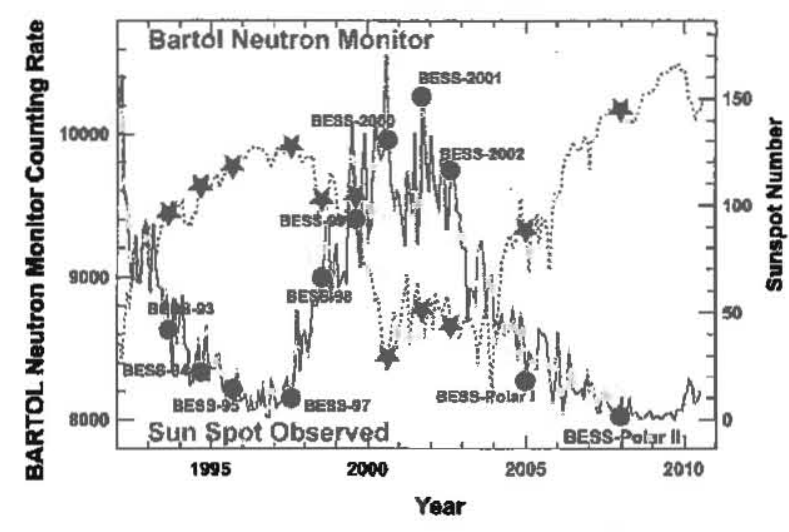

Fig. 2. Solar activity during the last BESS balloon flights.

the data expected from PAMELA in the BESS-Polar energy range. Most important, the BESS-Polar II flight took place very near solar minimum, as shown in Fig. 2, when sensitivity to a low-energy primary antiproton source is greatest. The long BESS-Polar II flight gave a $\sim 20$-fold increase in the number of antiprotons detected below $1 \mathrm{GeV}$ compared to the BESS-97 data at the previous solar minimum and a $\sim 14$-fold increase over the combined BESS-(95+97) data. After about one and two-thirds orbits of Antarctica, the BESS-Polar II flight was terminated over the West Antarctic Ice Sheet, as shown in Fig. 3, because of concerns over the flight trajectory. Logistics considerations prevented immediate recovery. Recovery of the BESSPolar II instrument was successfully carried out two years later in 2009-2010.

\section{Scientific progress from BESS-Polar observation}

The general BESS and BESS-Polar scientific progress has been reviewed in the references (Yamamoto, 2003;

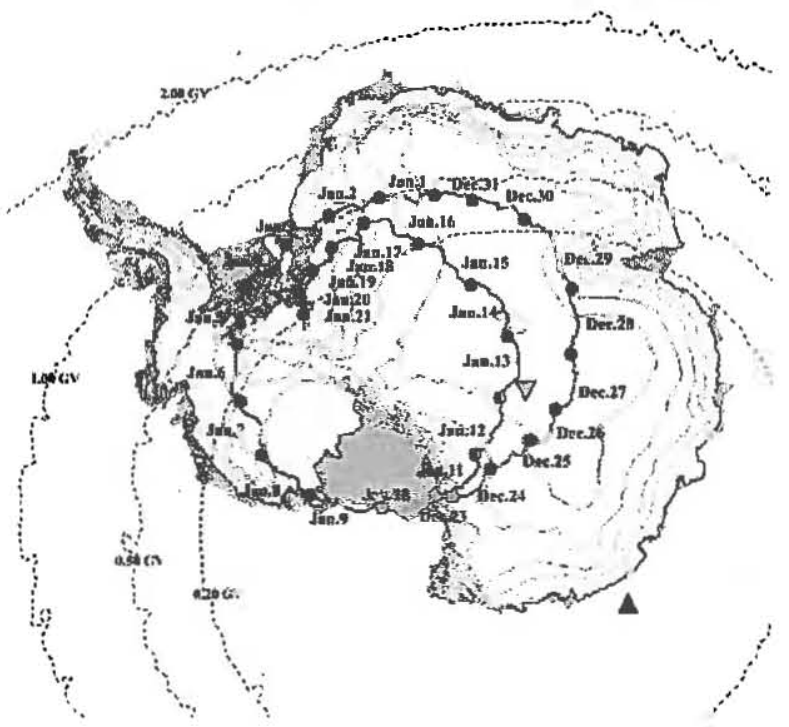

Fig. 3. Balloon flight trajectories in BESS-Polar II.
Mitchell et al., 2004, 2005; Yoshida et al., 2004; Yoshimura et al., 2008; Yamamoto et al., 2008; Mitchell et al., 2009). In this report, we focus on progress in the searches for cosmic-ray antiproton origins and for cosmological antimatter from the BESS-Polar program.

\subsection{Precise measurement of the antiproton spectrum}

Most cosmic-ray antiprotons are produced by interactions of high-energy Galactic cosmic rays with the interstellar medium. Due to production kinematics and to the energy spectra of the primary cosmic rays, the energy spectrum of these secondary antiprotons has a characteristic peak at around $2 \mathrm{GeV}$ and decreases sharply below and above the peak. This feature is clearly shown by the BESS data (Orito et al., 2000; Abe et al., 2008). Their mainly secondary origin makes antiprotons important tools to probe cosmic-ray transport as discussed in a recent comprehensive review (Strong et al., 2007). Deviations from the expected antiproton spectrum may signify the contribution of a primary source such as evaporation of primordial black holes (PBH) or annihilation of neutralino dark matter. PBH evaporation is expected to yield an antiproton spectrum with a peak well below $1 \mathrm{GeV}$. Superimposed on the steeply decreasing secondary antiproton spectrum, this could cause a flattening of the observed spectrum (Mitsui et al., 1996). Although the BESS $(95+97)$ antiproton flux measurements at the last solar minimum hint at an excess at low energy (Orito et al., 2000), successive measurements,

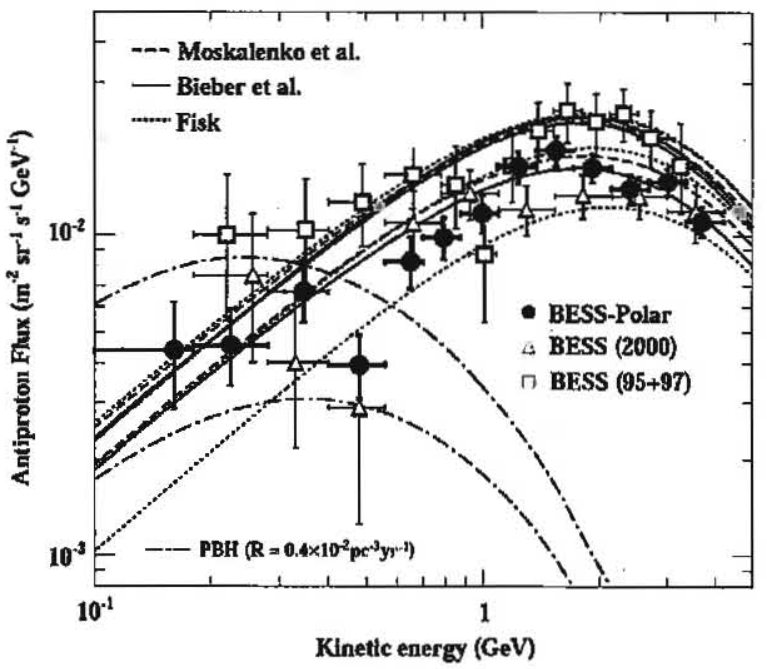

Fig. 4. Antiproton flux measured in BESS-Polar I and in previous BESS flights compared to secondary antiproton calculation with three models (Abe et al., 2008): the Standard Leaky Box (SLB) model modulated with a steady state drift model (solid curves: Bieber et al. (1999)) and the Diffusion plus Convection (DC) model modulated with a Heliospheric drift model (dashed curves: Moskalenko et al., 2002), and the DC model modulated with a spherical symmetric model (dotted curves: Fisk, 1971). The dash-dot curves are calculations of antiproton spectra from evaporation of primordial black holes with an explosion rate of $0.4 \times 10^{-2} \mathrm{pc}^{-3} \mathrm{yr}^{-1}$ modulated by $550 \mathrm{MV}$ (top: in 1995-1997) and $850 \mathrm{MV}$ (battom: 2004)

Please cite this article in press as: $Y$; nia:ruio, A., et al. Search for cosmic-ray antiproton ongins and for cosmological antimatter with BESS. J. Adv Space Res (2011), doi:10.1016/j.ast.2011.07.012 
taken after the solar minimum period, are more consistent witl a pure secondary nature.

Fig. 4 shows the antiproton spectrum measured by BESS-Polar I (Abe et al., 2008) compared with results from prerious BESS flights around solar minimum, 95+97 (Matsunaga et al., 1998; Orito et al., 2000) and maximum (Asioka et al., 2002), and compared with theoretical calculations. The solid curves are calculations of the interstellar secondary antiproton spectra from a Standard Leaky Box (SLB) model modulated with a steady state drift model (Bieber et al., 1999) in which the modulation is characterized by a tilt angle of the heliospheric current sheet and the Sun's magnetic polarity of (from top to bottom, and the first two are very close) $10^{\circ}(+), 10^{\circ}(-)$, and $70^{\circ}(-)$. The dashed curves are calculations with the Diffusion plus Corvection (DC) model of the secondary antiproton spectrum modulated with a Heliospheric drift model (Moskalenko et al., 2002; Moskalenko, 2006). The tilt angles, $10^{\circ}(+), 70^{\circ}(-)$, and $30^{\circ}(-)$ roughly correspond to the measurements with BESS (95+97), BESS (2000), and BESS-Polar I (2004), respectively (Zhao and Hoeksema, 1995; Hoeksema, 1995). The dotted curves are calculations with the DC model (Moskalenko et al., 2002) modulated with a standard spherically symmetric approach (Fisk, 1971 ), in which the modulation is characterized by a single parameter $(\phi)$ irrespective of the Sun's polarity. For each measurement, $\phi$ was obtained by fitting the corresponding proton spectrum measured by BESS, assuming the interstellar spectrum in (Orito et al, 2000). The values of $\phi$, $550 \mathrm{MV}, 1400 \mathrm{MV}$, and $850 \mathrm{MV}$ correspond to the measurements with BESS $(95+97)$, BESS (2000) and BESSPolar I (2004), respectively. The dash-dot curves are calculations of antiproton spectra from evaporation of PBH at a rate of $0.4 \times 10^{-2} \mathrm{pc}^{-3} \mathrm{yr}^{-1}$ (Maki et al., 1996; Yoshimura, 2001) modulated by a spherically symmetric approach.(Fisk, 1971) with modulation parameter $\phi$ independent of solar polarity. The expected signal from $\mathrm{PBH}$ evaporation is affected by solar modulation mcore than the secondary antiproton spectrum because of its low energy spectral peak. As might je expected, BESSPolar I antiproton measurements, taken during a trarsient period in advance of solar minimim, show no apparent excess, but provide a baseline secondary spectrum to be compared with the spectrum observed at solar minimum by BESS-Polar II.

The BESS-Polar II data analysis is still in progress. The full BESS-Polar II dataset is expected to yield $\sim 8000$ measured antiprotons. Fig. 5 shows particle identification plot with $\beta^{-1}$ versus rigidity using a quarter of the data from the BESS-Polar II. Fig. 6 shows a very preliminary antiproton energy spectrum from analysis, compared with the results from BESS-Polar I (2004) and BESS (95+97). The solid curves are calculations with the SLB model modulated with a steady state drift model (Bieber et al., 1999). The tilt angies of $10^{\circ}(+)$ and $30^{\circ}(-)$ approximately correspond to the measurements with BESS $(95+97)$ and BESS-Polar I (2004), respectively. The tilt angle during the BESS-Polar

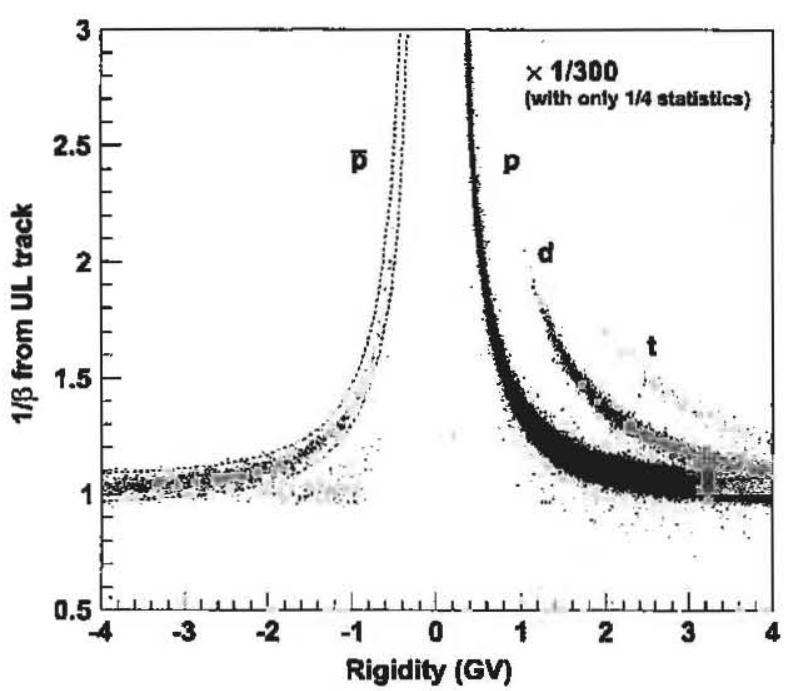

Fig. 5. The $\beta^{-1}$ versus rigidity plot, and antiproton selection band. For the negative rigidity, all the events with $R=-0.8 \mathrm{GV} / \mathrm{c}$ after Cherenkov veto cuts and JET $\mathrm{d} E / \mathrm{d} x$ cut are shown. For the positive rigidity, $0.1 \%$ of the events after Cherenkov veto cuts are shown.

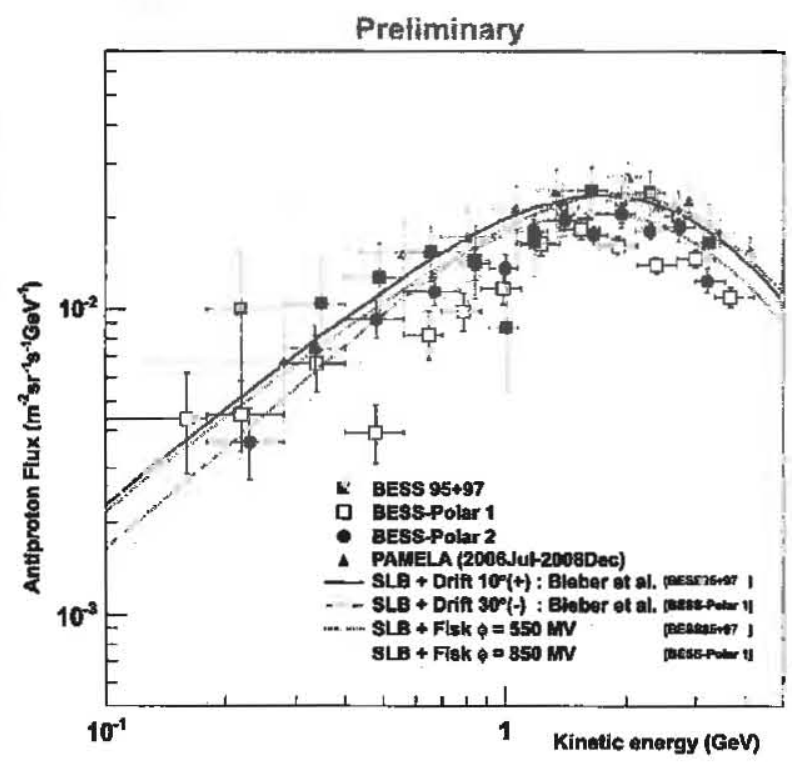

Fig. 6. Antiproton flux measured by BESS $(95+97)$, BESS-Polar I (2004), and a preliminary result by BESS-Polar II (2007-2008) which was obtained from the data analysis using a quarter observed events. The solid curves are secondary antiproton calculation with the SLB model modulated with the steady state drift model (Bieber et al.). The dotted curves are secondary antiproton calculations with the SLB model modulated with the spherically symmetric model (Fisk).

II flight would be about $10^{\circ}(-)$. The dashed curves are calculations with the SLB model modulated with the spherically symmetric approach (Fisk, 1971). The modulation parameters of $\phi=550 \mathrm{MV}$ and $850 \mathrm{MV}$ correspond to the measurements with BESS $(95+97)$ and BESS-Polar (2004), respectively. The modulation parameter for 


$\begin{aligned} & \text { JASR } 10669 \\ & 5 \text { August } 2011 \\ & \text { Disk Used }\end{aligned}$
$\begin{array}{ll}\text { A. Yamamoto et al / Advances in Space Research } x x x(2011) x x x-x x x & \text { No. of Pages 8, Model 5+ }\end{array}$

BESS-Polar II should be comparable to BESS $(95+97)$. As a preliminary result, the BESS-Polar II observation shows good consistency with the secondary antiproton calculations.

\subsection{Search for antihelium}

A fundamental question in cosmology is whether matter and entimatter are asymmetric or symmetric in the Universe. The Sakharov conditions of direct violation of baryon number conservation, $\mathrm{CP} \& \mathrm{C}$ symmetry breaking, and a period out of equilibrium in the very early Universe indicted a way to explain the apparent baryon domination observed (Sakharov, 1967). However, direct violation of baryon number conservation has never been demonstrated, and the strength of $\mathrm{CP}$ violations currently measured at accelerators are insufficient to explain strong matter/antimatter asymmetry. Detection of antihelium would provide direct evidence of antimatter domains in the Universe. Although antihelium might, in principle, be produced as seconcaries in cosmic-ray interactions, the resulting antihelium/telium ratio should be much less than $10^{-12}$ (Brown and Stecker, 1979).

The BESS-Polar-I experiment observed $8 \times 10^{6}$ helium events and no antihelium candidate was detected in the rigidity range 1-20 GV with an effective geometrical acceptance of $0.2 \mathrm{~m}^{2} \mathrm{sr}$. The resultant upper limit for the ratio of antihelium/helium was $4.4 \times 10^{-7}$. By accumulating all results from BESS through BESS-Polar I, an upper limit of $2.7 \times 10^{-7}$ was set in the rigidity range $1-14 \mathrm{GV}$ (Sasaki et al., 2008).

The BESS-Polar II experiment observed $4 \times 10^{7}$ helium events in a rigidity range of 1-14 GV with an effective geometrical acceptance of $0.3 \mathrm{~m}^{2} \mathrm{sr}$, and no antihelium candidate was detected. The resultant upper limit was $9.4 \times 10^{-8}$. By accumulating all results from BESS through BESS-Polar II, the $95 \%$ confidence level upper limit for antihelium/helium in the rigidity range $1-14 \mathrm{GV}$ has been reduced to be $6.9 \times 10^{-8}$ (Sasaki et al., 2010). Fig. 7 shows the BESS upper limits compared with other experiments. The upper limit for antihelium/helium has been reduced by two orders of magnitude compared to the first BESS limit (Ormes et al., 1997; Sasaki et al., 2002, 2008, 2010).

\section{Summary}

The BESS program has performed eleven scientific balloon flights successfully in northern Canada and Antarctica. It has aimed to search for cosmic-ray antiproton origins and for cosmological antimatter. The Antarctic flights of BESS-Polar I (2004) and BESS-Polar II (20072008) have yielded measurements of cosmic-ray antiprotons with unprecedented statistical accuracy and greatly increased the sensitivity of the antihelium search. The measurements made by BESS-Polar II took place near solar ninimum when sensitivity to a potential primary antiproton component at low energies is greatest. With

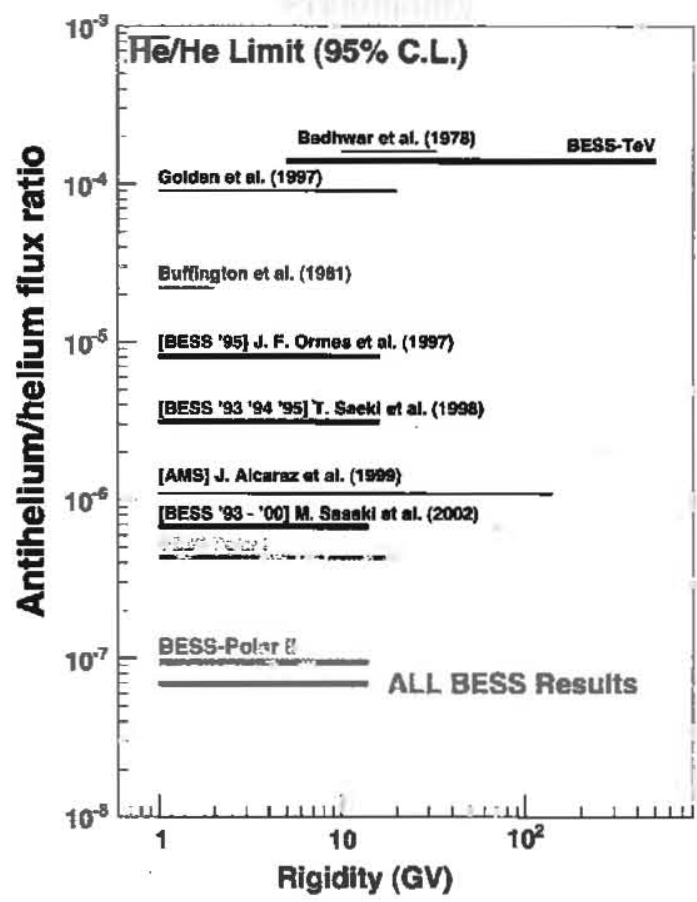

Fig. 7. Antihelium flux upper limits progressed in BESS and BESS-Polar experiments, compared with previous experiments (Sasaki et al., 2010).

statistics increased a factor of $>10$ compared to BESS measurements at the previous solar minimum, BESS-Polar II data shows good consistency with the secondary antiproton calculation. With further analysis, this data will place severe limits on any possible PBH evaporation contribution to the low-energy antiproton spectrum, and hence to limits on any possible density of primordial black holes. No antihelium candidate was observed in BESS through BESS-Polar II flight, and the $95 \%$ confidence level upper limit for antihelium/helium in the 1-14 GV rigidity range has been reduced to be $6.9 \times 10^{-8}$.

\section{Uncited-reforences}

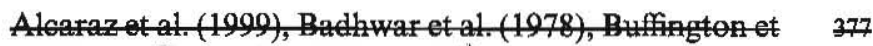
al. (1981), Sakk tai-(1998) (1994). Q3 378

\section{Acknowledgment}

The authors thank NASA Headquarters, ISAS/JAXA, GSFC, and KEK for continuous support and encouragement in the US-Japan cooperative BESS program. The authors also thank the NASA Balloon Program Office and the NASA Columbia Scientific Balloon Facility for their highly professional support of BESS conventional and long-duration balloon flight operations, and the National Science Foundation and Raytheon Polar Services Corporation for their support of the United States Antarctic Program. BESS-Polar is supported by the NASA 
Astrophysics Research and Analysis program in the US, and by the 'Kaken-hi' Grant in Aid, special promotion and fundamental research programs, MEXT, in Japan.

\section{References}

Abe, K., Fuke, H., Haino, S., et al. Measurement of the cosmic-ray lowenergy antiproton spectrum with the first BESS-Polar Antarctic flight. Phys. Lett. B 670, 103-108, 2008.

Ajima, Y., Anraku, K., Haga, T., et al. A superconducting solenoidal spectrometer for a balloon-borne experiment. Nucl. Instrum. Methods A 443, 71-100, 2000.

Alcaraz, J. et al. Search for antihelium in cosmic rays. Phys. Lett. B 461, 387-396, 1999.

Asaoka, Y., Shikaze, Y., Abe, K., et al. Measurement of cosmicray low energy antiproton and proton spectra in a transient period of solar field reversal. Phys. Rev. Lett. 88, 051101051104, 2002.

Badhwar, G.D., Golden, R.L., Lacy, J.L., et al. Relative abundance of antiprotons and antihelium in the primary cosmic radiation. Nature 274, 137-139, 1978

Barrau, A., Boudoul, G., Donato, F., et al. Antiproton from primordial black holes. Astron. Astrophys. 388, 676-687, 2002.

Bieber, J.W., Burger, R.A., Engel, R, et al. Antiprotons at solar maximum. Phys. Rev. Lett. 83, 674-677, 1999.

Brown, R.W., Stecker, F.W. Cosmological baryon-number domain structure from symmetry breaking in grand unified field theories. Phys. Rev. Lett. 43, 315-318, 1979.

Buffington, A., Schindler, S.M., Pennypacker, C.R. A measurement of the cosmic-ray antiproton flux and a search for antihelium. Astrophys. J. 248, 1179-1193, 1981.

Fisk, L.A. Solar modulation of galactic cosmic rays. J. Geophys. Res. 76, $221,1971$.

Hairo, S., Sanuki, T., Abe, K., et al. Measurements of primary and atmospheric cosmic-ray spectra with the BESS-TeV spectrometer. Phys. Lett. B 594, 35-46, 2004.

Hawking, S.W. Particle creation by black holes. Commun, Math. Phys. 43, 191, 1975.

Hoeksema, J.T. The large-scale structure of the heliospheric current sheet during the Ulysses epoch. Space Sci. Rev. 72, 137-148, 1995. See also, The Wilcox Solar Observatory http://wso.stanford.edu/ Tilts.html;

Maki, K., Mitsui, T., Orito, S. Local flux of low-energy antiprotons from evaporating primordial black holes. Phys. Rev. Lett. 76, 3474-3477, 1996.

Makida, Y., Kumazawa, T., Tanaka, K., et al. Performance of an ultrathin superconducting solenoid for particle astrophysics. IEEE Trans. Appl. Superconduct. 15 (2), 1248-1251, 2005.

Matsunaga, H., Orito, S., Matsumoto, H., et al. Measurement of low energy cosmic-ray antiprotons at solar minimum. Phys. Rev. Lett. 81, 4052-4055, 1998.

Mitchell, J.W., Abe, K., Anraku, K., et al, The BESS program. Nucl. Phys. B (Proc. Suppl.) 134, 31-38, 2004.

Mitchell, J.W., Abe, K., Anraku, K., et al. Precise measurements of the cosmic-ray antiproton spectrum with BESS including the effects of solar modulation. Adv. Space Res. 35 (1), 135, 2005.
Mitchell, J.W., Yamamoto, A., Yoshimura, K., et al. The BESS search for cosmic-ray antiproton origins and for cosmological antimatter, in: Proc. 31st ICRC (Lodz), 2009.

Mitsui, T., Maki, K., Orito, S. Phys. Lett. B 389, 169, 1996.

Moskalenko, I.V., Strong, A.W., Ormes, J.F., et al. Secondary antiprotons and propagation of cosmic rays in the galaxy and heliosphere. ApJ 565, 280, 2002.

Moskalenko, I.V. Private communication, 2006.

Orito, S., in: Proc. of ASTROMAG workshop, KEK Report, KEK-87, 1987.

Orito, S., Maeno, T., Matsunaga, H., et al. Precise measurement of cosmic-ray antiproton spectrum. Phys. Rev. Lett. 84, 1078-1081, 2000

Ormes, J.F. (Ed.), ASTRMAG. Report of the Astromag Definition Team, NASA, 1986.

Ormes, J.F., Moiseev, A.A., Saeki, T., et al. Antihelium in cosmic rays: a new upper limit and its significance. ApJ 482, L187-L190, 1997.

Saeki, T., Anraku, K., Orito, S., Ormes, J.F., et al. A new limit on the flux of cosmic antihelium. Phys. Lett. B 422, 319-324, 1998.

Sakharov, A.D. Violation of CP invariance, $\mathrm{C}$ asymmetry, and baryon asymmetry of the universe. JETP Lett. 5, 24-27, 1967.

Sasaki, M., Matsumoto, H., Nozaki, M., et al. Progress in search for antihelium with BESS. Nucl. Phys. B (Proc. Suppl.) 113, 202-207, 2002.

Sasaki, M., Haino, S., Abe, K., et a!. Search for antihelium: progress with BESS. Adv. Space. Res. 42 (3), 450-454, 2008.

Sasaki, M. Mitchell, J.W., Yamamoto, A., et al. Private communication, presented in European cosmic ray symposium, 2010.

Shikaze, Y., Orito, S., Mitsui, T., et al. Large-area scintillator hodoscope with 50 ps timing resolution onboard BESS. Nucl. Instrum. Methods A $455,596-606,2000$.

Strong, A.W., Moskalenko, I.V., Ptuskin, V.S. Cosmic ray propagation and interactions in the galaxy. Annu. Rev. Nucl. Part. Sci. 57, 285-327, 2007

Yamamoto, A., Mito, T., Haruyama, T., et al. Conceptual design of a thin superconducting soienoid for particle astrophysics. IEEE Trans. Mag. 24, 1421-1424, 1988.

Yamamoto, A., Anraku, K, Golden, R., et al. Balloon-borne experiment with a superconducting solenoid magnet spectrometer. Adv. Space Res. 14 (2), 75-87, 1994.

Yamamoto, A., Abe, K., Anraku, K., et al. BESS and its future prospect for polar long duration flights. Adv. Space Res. 30 (5), 1253-1262, $2002 a$.

Yamamoto, A., Makida, Y., Ohmiya, H., et al. A thin superconducting magnet for particle astrophysics. IEEE Trans. Appl. Superconduct. 12 (1), 438-441, 2002b.

Yamamoto, A. Latest results and future prospect, in: Proc. 28th ICRC (Tsukuba), 2003.

Yamamoto, A., Mitchell, J.W., Yoshimura, K, et al. Search for primordial antiparticles with BESS. Adv. Space Res. 42, 442-449, 2008.

Yoshida, T., Yamamoto, A., Mitchell, J.W., et al. BESS-Polar experiment. Adv. Space Res. 33 (10), 1775-1762, 2004.

Yoshimura, K. Cosmic-ray antiprotons and antinuclei. Adv. Space Res. 27 (4), 693-703, 2001.

Yoshimura, K., Abe, K., Fuke, H., et al. BESS-Polar experiment. Adv. Space Res. 42 (10), 1664-1669, 2008.

Zhao, X., Hoeksema, J.T. Predicting the heliospheric magnetic field using the current sheet-source surface model. Adv. Space Res. 16 (9), 181$184,1995$. 